\title{
Nucleus, Atom and the Universe - a combined study
}

\author{
U. V.S. Seshavatharam ${ }^{1^{*}}$, S. Lakshminarayana ${ }^{2}$ and B.V.S.T. Sai ${ }^{3}$ \\ ${ }^{1}$ Honorary faculty, I-SERVE, Alakapuri, Hyderabad-35, AP, INDIA. \\ ${ }^{2}$ Dept. of Nuclear Physics, Andhra University, Visakhapatnam-03, AP, INDIA. \\ ${ }^{3}$ Dept. of Mathematics and Comp. Science \& Engg, Guntur Engg. College, Guntur-19, AP, INDIA \\ *Corresponding author E-mail: seshavatharam.uvs@gmail.com
}

\begin{abstract}
Now a days many astrophysicists suggest that every galaxy constitutes a central (growing and fast spinning) black hole. Really it is amazing. If the observable universe constitutes so many galaxies then considering universe as a growing and light speed rotating black hole may not be far away from reality. It may be noted that role of dark energy in understanding the unification of fundamental forces is very insignificant. At any given cosmic time, Hubble length can be considered as the gravitational or electromagnetic interaction range. If light is coming from the atomic matter of the galaxy, then the observed redshift can be interpreted as an index of the galactic atomic 'light emission mechanism'. During cosmic evolution, as cosmic time increases, hydrogen atom emit photons with increased quanta of energy and thus past light quanta emitted from an old galaxy will have less energy and show a red shift with reference to our galaxy. During its journey light quanta will not lose energy and there will be no change in the light's wavelength. In this way without any difficulty the accelerating universe concept can be eliminated and a decelerating universe concept can be retained. To understand this light emission mechanism and to move further it is possible to consider the characteristic nuclear radius and characteristic angular momentum of the revolving electron as cosmological time oriented physical variables. Note that, in any bound system, 'operating force' only plays major role in maintaining the 'existence of the bound system' and 'angular momentum' is one of the result. If one is able to make the operating force as discrete, then automatically one can observe a discrete structure like discrete radii, discrete angular momentum and discrete energy levels. With reference to the classical force limit $\left(c^{4} / G\right)$ and considering Avogadro number $N$ as a large and discrete proportionality ratio, a characteristic discrete imaginary electroweak force magnitude can be defined as $\left(c^{4} / G\right) \div(n \cdot N)^{2}$ where $\mathrm{n}=1,2,3, .$.
\end{abstract}

Keywords: Hubble mass; Cosmic red shift; Cosmic thermal energy density; Classical force limit; Classical power limit; Avogadro number; Reduced Planck's constant; Imaginary electroweak force; Atomic gravitational constant; Light speed rotating black holes;

\section{Introduction}

It is accepted that a complete theory of the evolution of the universe will not be possible until the development of a successful quantum theory of gravity [1]. In this respect, cosmology resembles another branch of physics, the study of the elementary particles. By considering the observed 'cosmic redshift' as a result of galactic 'atomic light emission' mechanism the two central branches of physics i.e. 'cosmology' and 'quantum mechanics' can be studied in a unified manner. The outstanding problem in particle physics today is the inclusion of gravity in a single, unified quantum theory of all the fundamental interactions. Particle physicists have long suggested that the four fundamental forces of nature (viz. the gravitational, electromagnetic, weak nuclear and strong nuclear forces) are separate, low energy manifestations of what was once a single force at times close to the Big Bang. It is postulated that as the universe expanded and cooled, this single force gradually broke down into the four separate interactions observed today. A detailed quantum theory that describes the electromagnetic and weak nuclear forces in terms of a single force (the electroweak interaction) was developed in the 1970's and dramatically verified by high-energy experiments in particle accelerators a decade later. More ambitious quantum theories that incorporate the strong nuclear force (Grand Unified Theories) have been developed, and some experimental support for these models has emerged. However, unification theories that seek to unify the force of gravity with all the other forces (Theories of Everything) remain elusive, as the gravitational interaction lacks a quantum formulation. 
To unify cosmology, quantum mechanics and the four observed fundamental cosmological interactions - certainly a 'unified force' is required. In this connection $\left(c^{4} / G\right)$ can be considered as the fundamental unified force or the classical force limit. Similarly $\left(c^{5} / G\right)$ can be considered as the classical power limit. Please note that, in any bound system, 'operating force' only plays major role in maintaining the 'existence of the bound system' and 'angular momentum' is one of the result. By any reason and with any 'large proportionality ratio', if one is able to bring down its magnitude to the observed force magnitudes as in atomic system and make it discrete, then automatically one can see a discrete structure or arrangement in the atomic system. Then the observed discrete radii, discrete angular momentum and discrete energy levels can be easily understood. Now the fundamental question to be answered is - How to select a 'discrete' and 'large' proportionality ratio? Answer is very simple. To implement the existing large numbers and to see the consequences.

Many physicists think about the possible variation of the 'fine structure ratio' and experiments are in progress. In a theoretical approach, a varying $\alpha$ has been proposed as a characteristic and unified way of solving the fundamental problems in cosmology and astrophysics. More recently, theoretical interest in varying constants (not just $\alpha$ ) has been motivated by string theory and other such proposals for going beyond the Standard Model of particle physics. In October 2011 Webb et. al. [2] reported a variation in $\alpha$ dependent on both 'redshift' and 'spatial direction'. Here it should be noted that, the concept - 'variation of alpha' directly and indirectly is giving a clue to think about the possible 'variation' of the reduced Planck's constant or Planck's constant. This is a very sensitive point and needs strong experimental evidence and vigorous theoretical analysis.

In understanding the basic concepts of unification or TOE, role of dark energy and dark matter is insignificant. Even though there were a number of papers/books published on cosmology, the attempt for a comprehensive study on this subject, coupled with comparative studies with the modern cosmology on one hand and with the modern atomic physics on the other, was not made by anybody so far. The present study can be considered as a 'beginning project' in this field. It is an interesting part of the study of cosmology and fundamental interactions. So far no Institute has taken this subject for R\&D. This idea is quite unique, natural and the openness in the subjects of cosmology and fundamental interactions can be eliminated.

\subsection{Edwin Hubble's opinion on Cosmic redshift}

In 1947 Hubble [4] suggested that "The red shifts are more easily interpreted as evidence of motion in the line of sight away from the earth - as evidence that the nebulae in all directions are rushing away from us and that the farther away they are, the faster they are receding. This interpretation lends itself directly to theories of expanding universe. The interpretation is not universally accepted, but even the most cautious of us admit that red shifts are evidence of either an expanding universe or of some hitherto unknown principle of nature".

"Attempts have been made to attain the necessary precision with the $100 \mathrm{inch}$, and the results appear to be significant. If they are valid, it seems likely that the red-shifts may not be due to an expanding universe, and much of the current speculation on the structure of the universe may require re-examination. The significant data, however, were necessarily obtained at the very limit of a single instrument, and there were no possible means of checking the results by independent evidence. Therefore the results must be accepted for the present as suggestive rather than definitive".

"We may predict with confidence that the 200 inch will tell us whether the red shifts must be accepted as evidence of a rapidly expanding universe, or attributed to some new principle in nature. Whatever may be the answer, the result may be welcomed as another major contribution to the exploration of the universe."

\subsection{Albert Einstein's opinion on unification of electromagnetic and gravitational interactions}

Note that, Einstein, more than any other physicist, untroubled by either quantum uncertainty or classical complexity, believed in the possibility of a complete, perhaps final, theory of everything. [5]. He also believed that the fundamental laws and principles that would embody such a theory would be simple, powerful and beautiful. Physicists are an ambitious lot, but Einstein was the most ambitious of all. His demands of a fundamental theory were extremely strong. If a theory contained any arbitrary features or undetermined parameters then it was deficient, and the deficiency pointed the way to a deeper and more profound and more predictive theory. There should be no free parameters - no arbitrariness. According to his philosophy, electromagnetism must be unified with general relativity, so that one could not simply imagine that it did not exist. Furthermore, the existence of matter, the mass and the charge of the electron and the proton (the only elementary particles recognized back in the 1920's), were arbitrary features. One of the main goals of a unified theory should be to explain the existence and calculate the properties of matter. 


\subsection{Major role of Mach's principle in understanding the universe and the atom}

In between the 'flat' universe and the 'closed' universe, there is one compromise. That is 'Hubble volume'. Without considering the Mach's principle, physical meaning cannot be attached to "Hubble volume". Note that till today quantitatively Mach's principle [6] was not implemented successfully in cosmic and nuclear physics. If we do not yet know whether the universe is spatially closed or open, then the idea of 'Hubble volume' can be used as a tool in cosmology and unification. Some cosmologists use the term 'Hubble volume' to refer to the volume of the observable universe. At any cosmic time, if "Hubble mass" is the product of cosmic critical density and the Hubble volume, then it can be suggested that, "within the Hubble volume, each and every point in free space is influenced by the Hubble mass". It seems to be a quantitative description to the Mach's principle. Now a days many astrophysicists assume that every galaxy constitutes a cental (growing and fast spinning) black hole. Really it is a very strange and very interesting concept. It is true that the observable universe constitutes so many galaxies. If so considering universe as a growing and light speed rotating black hole may not be far away from reality. In the universe, if the current critical density is $\left(\rho_{c}\right)_{0} \cong\left(3 H_{0}^{2} / 8 \pi G\right)$ and the current characteristic Hubble radius is $R_{0} \cong\left(c / H_{0}\right)$, characteristic current mass of the cosmic Hubble volume is $M_{0} \cong\left(c^{3} / 2 G H_{0}\right)$ and its 'Schwarzschild radius' resembles the 'Hubble length'. For the time being let us call this mass as 'Hubble mass'. With this definition, apart from cosmology, Mach's principle can be given a fundamental and unified significance in atomic, nuclear and particle physics!

\subsection{The cosmic 'critical density' and its dimensional analysis}

Recent findings from the University of Michigan suggest that the shape of the Big Bang might be more complicated than previously thought, and that the early universe spun on an axis. A left-handed and right-handed imprint on the sky as reportedly revealed by galaxy rotation would imply the universe was rotating from the very beginning and retained an overwhelmingly strong angular momentum. Galaxies spin, stars spin, and planets spin. So, why not the whole universe? The consequences of a spinning universe seem to be profound, natural and 'cosmic collapse' can be prevented. Thus 'cosmic (light speed) rotation' can be considered as an alternative to the famous 'repulsive gravity' concept.

With a simple derivation it is possible to show that, Hubble's constant $\left(H_{t}\right)$ represents cosmological angular velocity. Assume that, a planet of mass $(\mathrm{M})$ and radius $(\mathrm{R})$ rotates with angular velocity $\left(\omega_{e}\right)$ and linear velocity $\left(v_{e}\right)$ in such a way that, free or loosely bound particle of mass $(\mathrm{m})$ lying on its equator gains a kinetic energy equal to potential energy as,

$$
\begin{aligned}
& \frac{1}{2} m v_{e}^{2}=\frac{G M m}{R} \\
& R \omega_{e}=v_{e}=\sqrt{\frac{2 G M}{R}} \text { and } \omega_{e}=\frac{v_{e}}{R}=\sqrt{\frac{2 G M}{R^{3}}}
\end{aligned}
$$

i.e linear velocity of planet's rotation is equal to free particle's escape velocity. Without any external power or energy, test particle gains escape velocity by virtue of planet's rotation. Using this idea, 'Black hole radiation' and 'origin of cosmic rays' can be understood. Note that if Earth completes one rotation in one hour then free particles lying on the equator will get escape velocity. Now writing, $M=\frac{4 \pi}{3} R^{3} \rho_{e}$,

$\omega_{e}=\frac{v_{e}}{R}=\sqrt{\frac{8 \pi G \rho_{e}}{3}}$ Or $\omega_{e}^{2}=\frac{8 \pi G \rho_{e}}{3}$

Density, $\rho_{\mathrm{e}}=\frac{3 \omega_{\mathrm{e}}^{2}}{8 \pi \mathrm{G}}$

In real time, this obtained density may or may not be equal to the actual density. But the ratio, $\frac{8 \pi G \rho_{\text {real }}}{3 \omega_{\text {real }}^{2}}$ may have some physical meaning. The most important point to be noted here, is that, as far as dimensions and units are considered, from equation (4), it is very clear that, proportionality constant being $\frac{3}{8 \pi G}$,

density $\propto(\text { angular velocity })^{2}$ 
Equation (4) is similar to "flat model concept" of cosmic "critical density"

$\rho_{c}=\frac{3 H_{t}^{2}}{8 \pi G}$

Comparing equations (4) and (6) dimensionally and conceptually, i.e.

$\rho_{e}=\frac{3 \omega_{e}^{2}}{8 \pi G}$ with $\rho_{\mathrm{c}}=\frac{3 H_{\mathrm{t}}^{2}}{8 \pi \mathrm{G}}$

$H_{t}^{2} \rightarrow \omega_{\mathrm{e}}^{2}$ and $H_{t} \rightarrow \omega_{e}$

It is very clear that, dimensions of 'Hubble's constant' must be 'radian/second'. In any physical system under study, for any one 'simple physical parameter' there will not be two different units and there will not be two different physical meanings. This is a simple clue and brings "cosmic rotation" into picture. This is possible in a closed universe only. Cosmic models that depend on this "critical density" may consider 'angular velocity of the universe' in the place of 'Hubble's constant'. In the sense, 'cosmic rotation' can be included in the existing models of cosmology.

\section{Possible assumptions in unified cosmic physics}

Possible assumptions in unified cosmic physics can be expressed in the following way [7]:

A) Hubble length $\left(c / H_{t}\right)$ can be considered as the gravitational or electromagnetic interaction range.

B) Being a primordial evolving black hole and angular velocity being $H_{t}$, universe is always rotating with light speed.

C) The key conceptual link that connects the gravitational and non-gravitational forces is - the classical force limit

$$
F_{C} \cong \frac{c^{4}}{G} \cong 1.2106 \times 10^{44} \text { newton }
$$

It can be considered as the unified cosmic force magnitude or the upper limit of the string tension. In its inverse form it appears in Einstein's theory of gravitation as $\frac{8 \pi G}{c^{4}}$. It has multiple applications in Black hole physics and Planck scale physics. It has to be measured either from the experiments or from the cosmic and astronomical observations.

D) Ratio of 'classical force limit $\left(F_{C}\right)$ ' and 'imaginary electroweak force magnitude $\left(F_{I}\right)$ ' is $N^{2}$ where $N$ is a large number close to the Avogadro number.

$\frac{F_{C}}{F_{I}} \cong N^{2} \cong \frac{\text { Upper limit of classical force }}{\text { Imaginary electroweak force magnitude }}$

Thus the proposed imaginary electroweak force magnitude is $F_{I} \cong 3.33715 \times 10^{-4}$ newton. It seems to be more fundamental than the electroweak and strong nuclear forces. It plays a very interesting role in understanding the scattering distance between electron and the charged nucleus. Along with the characteristic nuclear size, this force plays a vital role in understanding the revolving electron's distance from the nucleus in the hydrogen atom. Considering this $F_{I}$ 'charged', Higgs fermion and boson masses can be fitted. Muon and tau masses can be fitted accurately [7].

With $\ln \left(\frac{F_{C}}{F_{I}}\right) \cong \ln \left(N^{2}\right)$, proton-electron mass ratio can be fitted. Gravitational constant or the Avogadro number can be fitted [7].

E) Avogadro number is discrete and thus the imaginary electroweak force is discrete. The discrete imaginary electroweak force can be expressed as

$$
\frac{c^{4}}{(n \cdot N)^{2} G} \cong \frac{c^{4}}{n^{2} \cdot\left(N^{2} G\right)} \cong \frac{c^{4}}{n^{2} \cdot G_{A}} \cong \frac{F_{I}}{n^{2}}
$$

where $n=1,2,3, .$. This discrete imaginary electroweak force may be the responsible force for revolving electron's discrete total energy in hydrogen atom.

F) Atomic gravitational constant can be represented as squared Avogadro number times the classical gravitational constant. Thus,

$G_{A} \cong N^{2} G$ 
where ' $G_{A}$ ' is the Atomic gravitational constant, ' $N$ ' is the Avogadro number and ' $G$ ' is the classical gravitational constant.

G) Characteristic nuclear radius increases with cosmic time. Please see section 3.5.

H) Reduced Planck's constant or the characteristic angular momentum of the revolving electron increases with cosmic time. At present if we represent $\hbar \cong \hbar_{0}$, in the past the operating reduced Planck's constant was $\mathrm{h}_{t}$ and its magnitude was less than $\hbar_{0}$. Please see section 3.6.

Thus at any given cosmic time $t$,

1) $\frac{d(\hbar)}{d t}$ is a measure of cosmic rate of expansion. As time is passing, one can expect a very small change in $\frac{d(\hbar)}{d t}$ and it may be beyond the scope of experimental accuracy. But to have a rapid (detectable) change in $\frac{d(\hbar)}{d t}$ present cosmic time should run fast or should accelerate. It is possible to show that, potential energy of electron in hydrogen atom is directly proportional to $\hbar^{2}$. Bohr's second postulate which suggests that potential energy of electron in hydrogen atom is inversely proportional to $\hbar^{2}$ seems to be a coincidence.

2) During cosmic evolution, as cosmic time increases, hydrogen atom emit photons with increased quanta of energy. Thus past light quanta emitted from old galaxy will have less energy and show a red shift with reference to our galaxy. During journey light quanta will not lose energy and there will be no change in light wavelength.

3) It may be noted that, increased redshifts and increased distances forced Edwin Hubble to propose the Hubble's law. With reference to our laboratory or our galaxy, the basic or original definition of present/current redshift $\left(z_{0}\right)$ seems to be:

$z_{0} \cong \frac{E_{0}-E_{G}}{E_{0}} \cong \frac{\lambda_{G}-\lambda_{0}}{\lambda_{G}} \leq 1$

But not $z_{0} \cong \frac{E_{0}-E_{G}}{E_{G}} \cong \frac{\lambda_{G}-\lambda_{0}}{\lambda_{0}}$.

Here $E_{0} \cong \frac{h c}{\lambda_{0}}$ is the energy of photon at our galaxy/laboratory and $E_{G} \cong \frac{h c}{\lambda_{G}}$ is the energy of photon at the observed galaxy when it was emitted. Similarly $\lambda_{G}$ is the wave length of light received from observed galaxy and $\lambda_{0}$ is the wave length of light in laboratory. Even though both relations are ad-hoc and not absolute definitions, compared to relation (14), relation (13) seems to be some what reliable. Note that, based on the increasing value of the Planck's constant, present red shift $\left(z_{0}\right)$ will be directly proportional to age difference between our galaxy and observed galaxy or time taken by light to reach our galaxy from the old galaxy $(\Delta t)$. Thus $z_{0} \propto \Delta t$ and

$z_{0} \cong H_{0} \Delta t$.

Here $H_{0}$ is the proportionality constant. In this way $H_{0}$ can be incorporated directly. Time taken by light to reach our galaxy or the age difference of our galaxy and observed galaxy can be expressed as,

$\Delta t \cong \frac{z_{0}}{H_{0}}$.

$c \Delta t \cong z_{0} \cdot \frac{c}{H_{0}}$.

In this way, the basic and original definition of 'galaxy receding' and 'accelerating universe' [8] concepts can be eliminated and a 'decelerating or expanded universe' concept can be continued without any difficulty. Now the fundamental question to be answered is: If $\left(\hbar_{t}\right)$ takes the role of $(\hbar)$, how to define the red shift? In section 3.7, considering $\left(\frac{E_{0}-E_{G}}{E_{0}}\right)$ we proposed a simple solution to this problem. With different galaxies and with different $(\Delta t)$, $H_{0} \cong\left(\frac{z_{0}}{\Delta t}\right)_{G_{1}} \cong\left(\frac{z_{0}}{\Delta t}\right)_{G_{2}} \cong\left(\frac{z_{0}}{\Delta t}\right)_{G_{3}}$

where $G_{1}, G_{2}$ and $G_{3}, .$. represent different galaxies. If it is possible to show that, (from the observer) older galaxy's distance increases with its 'age', then automatically the concept of 'galaxy receding' and 'accelerating universe' concept can be put for a revision at fundamental level. 
4) At any given cosmic time, the Schwarzschild radius of universe is

$\frac{2 G M_{t}}{c^{2}} \cong \frac{c}{H_{t}}$

where $M_{t}$ is the cosmic mass at that time. With this idea, at any given cosmic time, cosmic size can be constrained to a maximum instead of infinity. The cosmic mass can be expressed as

$M_{t} \cong \frac{c^{3}}{2 G H_{t}}$.

It can be called as the 'Hubble mass'. Thus the cosmic volume density takes the following well known 'critical density' form,

$\left(\rho_{v}\right)_{t} \cong \frac{c^{3}}{2 G H_{t}} \div \frac{4 \pi}{3}\left(\frac{c}{H_{t}}\right)^{3} \cong \frac{3 H_{t}^{2}}{8 \pi G}$.

It can be called as the cosmic Hubble density.

\section{Applications of the proposed (possible) assumptions}

\subsection{About the new fundamental mass unit}

Alternative to the Planck mass and with reference to the fundamental physical constants ( $e$ and $\mathrm{G})$, a fundamental mass unit can be constructed as $\left(M_{e}\right)^{ \pm} \cong \sqrt{\frac{e^{2}}{4 \pi \varepsilon_{0} G}} \cong 1.859211 \times 10^{-9} \mathrm{~kg}$. It can be considered as a characteristic fundamental unified charged mass unit. It is noticed that, the ratio $\left(\frac{M_{t}}{M_{e}}\right)$ plays a very interesting role in fitting the cosmic matter density and thermal energy density.

\subsection{Cosmic Thermal Energy Density}

At any given cosmic time, ratio of cosmic volume energy density and cosmic thermal energy density can be expressed as

$$
\left(\frac{\rho_{v} c^{2}}{a T^{4}}\right)_{t} \cong\left[1+\ln \left(\frac{M_{t}}{M_{e}}\right)\right]^{2}
$$

Thus independent of the radiation constants, at any given cosmic time, (equivalent) cosmic thermal energy density can be expressed as

$a T_{t}^{4} \cong\left[1+\ln \left(\frac{M_{t}}{M_{e}}\right)\right]^{-2}\left(\frac{3 H_{t}^{2} c^{2}}{8 \pi G}\right)$

If $H_{0}$ is close to $71 \mathrm{~km} / \mathrm{sec} / \mathrm{Mpc}$, obtained thermal energy density is $4.161 \times 10^{-14} \mathrm{~J} / \mathrm{met}^{3}$. For a junior cosmologist this is a miracle. For a senior cosmologist this is a big question mark. For a neutral cosmologist this is a fine tool. For a mathematician this is a tricky job and for a thermal engineer this is very exciting (why because (s)he is not giving any thermal input). Any how this is the time to resolve the issue and future will definitely take care of this strange coincidence. When, $M_{t} \cong M_{e} \Rightarrow a T^{4} \cong \rho_{v} c^{2}$. At any given cosmic time, cosmic radius can be expressed as

$$
\frac{c}{H_{t}} \cong \exp \left(\sqrt{\left(\frac{\rho_{v} c^{2}}{a T^{4}}\right)_{t}}-1\right) \cdot \frac{2 G M_{e}}{c^{2}}
$$

\subsection{Cosmic matter density}

Approximately relation between cosmic volume density $\left(\rho_{v}\right)_{t}$ and matter density $\left(\rho_{m}\right)_{t}$ can be expressed as 


$$
\left(\rho_{m}\right)_{t} \cong\left[1+\ln \left(\frac{M_{t}}{M_{e}}\right)\right]^{-1}\left(\frac{3 H_{t}^{2}}{8 \pi G}\right)
$$

At any given cosmic time, cosmic matter energy density can be considered as the geometric mean of cosmic volume energy density and cosmic thermal energy density. Note that, at present obtained matter density $\rho_{m}$ can be compared with the elliptical and spiral galaxy matter density. Based on the average mass-to-light ratio for any galaxy

$\left(\rho_{m}\right)_{0} \cong 1.5 \times 10^{-32} \eta h_{0}$ gram $/ \mathrm{cm}^{3}$

where for any galaxy, $\langle M / L\rangle_{\text {Galaxy }}=\eta\langle M / L\rangle_{\text {Sun }}$ and the number: $h_{0} \cong \frac{H_{0}}{100 \mathrm{Km} / \mathrm{sec} / \mathrm{Mpc}} \cong \frac{71}{100} \cong 0.71$. Note that almost $80 \%$ of the galaxies are in the form of elliptical and spiral galaxies. For spiral galaxies, $\eta h_{0}^{-1} \cong 9 \pm 1$ and for elliptical galaxies, $\eta h_{0}^{-1} \cong 10 \pm 2$. For our galaxy inner part, $\eta h_{0}{ }^{-1} \cong 6 \pm 2$. Thus the average $\eta h_{0}^{-1}$ is very close to 8 to 9 and its corresponding matter density is $(6.05$ to 6.8$) \times 10^{-32} \mathrm{gram} / \mathrm{cm}^{3}$.

\subsection{The cosmological fine structure ratio}

If $\left(\rho_{v} c^{2}\right)_{0}$ is the present cosmic volume energy density, $a T_{0}^{4}$ is the present cosmic thermal energy density and $\alpha$ is the present cosmological fine structure ratio, it is noticed that,

$\ln \sqrt{\left(\frac{a T^{4}}{\rho_{v} c^{2}}\right)_{0} \cdot \frac{4 \pi \varepsilon_{0} G M_{0}^{2}}{e^{2}}} \cong\left(\frac{1}{\alpha}\right) \cong\left(\frac{1}{\alpha}\right)_{0}$

From relation (22)

$\ln \left\{\left[1+\ln \left(\frac{M_{0}}{M_{e}}\right)\right]^{-1} \cdot \frac{M_{0}}{M_{e}}\right\} \cong\left(\frac{1}{\alpha}\right)_{0}$

This application can be considered as a key tool in particle cosmology. Note that large dimensionless constants and compound physical constants reflect an intrinsic property of nature. In this way, in a unified manner, the present fine structure ratio can be fitted. From this relation it is possible to say that, cosmological rate of change in fine structure ratio, $\frac{d}{d t}\left(\frac{1}{\alpha}\right)$ may be considered as an index of the future cosmic acceleration. As time is passing, one can expect a very small change in $\frac{d}{d t}\left(\frac{1}{\alpha}\right)$ and it may be beyond the scope of experimental accuracy. But to have a rapid (detectable) change in $\frac{d}{d t}\left(\frac{1}{\alpha}\right)$, present cosmic time should run fast or should accelerate . At any given cosmic time, it can be expressed as

$\ln \left\{\left[1+\ln \left(\frac{M_{t}}{M_{e}}\right)\right]^{-1} \cdot \frac{M_{t}}{M_{e}}\right\} \cong\left(\frac{1}{\alpha}\right)_{t}$

If $M_{t} \rightarrow M_{e},\left(\frac{1}{\alpha}\right)_{t} \rightarrow 0$

\subsection{Current or present characteristic nuclear radius}

The nucleus can be modeled as a sphere of positive charge for the interpretation of electron scattering experiments: because there is no definite boundary to the nucleus, the electrons 'see' a range of cross-sections, for which a mean can be taken. The qualification of "rms" (for "root mean square") arises because it is the nuclear cross-section, proportional to the square of the radius, which is determining for electron scattering. The first estimate of a nuclear charge radius was made by Hans Geiger and Ernest Marsden in 1909, under the direction of Ernest Rutherford at the Physical 
Laboratories of the University of Manchester, UK [9]. Modern direct measurements are based on the scattering of electrons by nuclei. With reference to the current mass $M_{0} \cong\left(c^{3} / 2 G H_{0}\right)$ of the expanding black hole universe, we discovered a very strange relation and it can be expressed in the following way.

$\left(R_{N}\right)_{0} \cong \frac{G \sqrt{M_{0} \sqrt{m_{p} m_{e}}}}{c^{2}} \cong 1.37 \mathrm{fm}$

This length can be considered as the current characteristic nuclear size in the current expanding universe. This is a remarkable coincidence and seems to open a new window in 'cosmology' and 'nuclear physics'. Note that, $1.4 \mathrm{fm}$ is nothing but the observed and well understood strong interaction range. The most important point is that, as the black hole universe is expanding, from nuclear physics point of view, its expansion rate can be verified with $\frac{d}{d t}\left(R_{N}\right)_{t}$. As time is passing, one can expect a very small change in $\frac{d}{d t}\left(R_{N}\right)$ and it may be beyond the scope of experimental accuracy. But to have a rapid (detectable) change in $\frac{d}{d t}\left(R_{N}\right)$ present cosmic time should run fast or should accelerate. In this regard the proposed basic idea is that, the characteristic nuclear size that is measured in electron scattering experiments increases with increasing cosmic size. We propose the following (another) discovered relation and it has to be derived with a suitable model.

$$
\left(R_{x}\right)_{0} \cong\left(\frac{F_{I}}{F_{C}}\right)\left(\frac{m_{p}}{m_{e}}\right)^{2}\left(\frac{c}{H_{0}}\right) \cong\left(\frac{\hbar_{0} c}{G_{A} m_{e}^{2}}\right)^{2} \frac{2 G_{A} m_{e}}{c^{2}} \cong 1.21 \text { to } 1.22 \mathbf{f m}
$$

Another interesting observation is $\frac{\left(R_{x}\right)_{0}}{2} ; 0.61 \mathrm{fm} \cong\left(R_{y}\right)_{0}$ can be considered as the characteristic imaginary electroweak force range and $\frac{\left(R_{x}\right)_{0}}{\sqrt{2}} \cong 0.86 \mathrm{fm}$ can be compared with the rms radius of proton. At utmost fundamental level, it may not be possible to give a proof or derivation for any new discovered relation. In due course, it can be applied in different ways and its validity can be verified. From one point of view, it is very peculiar and from another point of view it is very complicated to interpret. Why because till today no model (including the famous string theory) could explain the origin of the strong interaction range! Whether to 'consider this relation' or 'discard this relation', depends only on our choice of scientific interest. Authors noticed interesting applications of this characteristic nuclear size in atomic physics and electroweak interaction [7].

\subsection{The Cosmological Reduced Planck's Constant}

From above relations it can be guessed that, there exists a strong interconnection in between universe and the Hydrogen atom. It should be noted that, in Bohr's theory of hydrogen atom, neither the nuclear mass nor the nuclear size has been considered for understanding the energy spectra of the excited hydrogen atom. Not only that, till today no one knows the origin of the well understood Planck's constant. In this connection the following expression can be considered as a discovery.

$$
n . \hbar_{0} \cong m_{e} c^{2} \sqrt{\frac{m_{e}\left(R_{y}\right)_{0}^{2}}{\left(\frac{F_{I}}{n^{2}}\right)\left(R_{y}\right)_{0}}}
$$

Here $m_{e}\left(R_{y}\right)_{0}^{2}$ can be considered as the imaginary moment of inertia of electron about its axis of rotation, where the radial distance is $\left(R_{y}\right)_{0} ; 0.61 \mathrm{fm} .\left(\frac{F_{I}}{n^{2}}\right)\left(R_{y}\right)_{0}$ can be considered as the characteristic work done related with the discrete imaginary electroweak force and $n=1,2,3, .$. Above expressions' simple form is:

$$
n \cdot \hbar_{0} \cong \sqrt{\left(n^{2} \cdot G_{A}\right) m_{e}^{3}\left(R_{y}\right)_{0}}
$$

From relation (32), this relation takes the form.

$$
n . \hbar_{0} \cong \frac{n \cdot G m_{p} \sqrt{M_{0} m_{e}}}{c} \cong \sqrt{\frac{M_{0}}{m_{e}}} \cdot \frac{n \cdot G m_{p} m_{e}}{c}
$$


Here $\left(M_{0} / m_{e}\right)$ can be considered as the number of electrons in the present universe of mass, $M_{0} \cong\left(c^{3} / 2 G H_{0}\right)$. As a physicist or cosmologist one must make an attempt to understand this strange coincidence. Here, the authors request the valuable views of Einstein on unification of 'gravity', 'electromagnetism' and 'quantum mechanics' be reconsidered. If an electron revolves around a proton of size close to its 'rms' radius and (electron \& proton) are the massive elementary atomic particles of the observable expanding universe, then the above relation may be given some consideration in the unification program. If so, present Hubble's constant can be expressed as

$H_{0} \cong \frac{G m_{p}^{2} m_{e} c}{2 \hbar_{0}^{2}} \cong 70.743 \mathrm{~km} / \mathrm{sec} / \mathrm{Mpc}$

Thus it is possible to guess that,

$H_{0} \hbar_{0}^{2} \cong H_{t} \hbar_{t}^{2} \cong \frac{G m_{p}^{2} m_{e} c}{2} \cong$ constant

Now here we are presenting the following interesting relation.

$\left(\frac{\hbar_{0} c}{G_{A} m_{e}^{2}}\right)^{2} \cong \frac{F_{I}\left(R_{y}\right)_{0}}{m_{e} c^{2}}$

Substituting the proposed expression for ${ }^{`} h_{0}{ }^{\prime}$ from relation (32), in this relation RHS can be obtained.

\subsection{Electron's Characteristic Potential Energy and the cosmic red shift}

With reference to the proposed ' $F_{I}$ ' and $\left(R_{y}\right)_{0}$ present Bohr radius can be expressed as

$\left(a_{0}\right)_{0} \cong\left(\frac{m_{e} c^{2}}{F_{I}\left(R_{y}\right)_{0}}\right) \cdot \frac{e^{2}}{4 \pi \varepsilon_{0} \cdot \frac{1}{2} \sqrt{m_{p} m_{e}} \cdot c^{2}}$

This is one interesting observation or discovery and is a remarkable coincidence. Clearly speaking, as the universe is expanding or evolving, within the atom, characteristic nuclear size is increasing and distance between electron and the nucleus is decreasing. Now the discrete Bohr radii can be expressed as

$n^{2}\left(a_{0}\right)_{0} \cong\left(\left(\frac{n^{2}}{F_{I}}\right) \frac{m_{e} c^{2}}{\left(R_{y}\right)_{0}}\right) \cdot \frac{e^{2}}{4 \pi \varepsilon_{0} \cdot \frac{1}{2} \sqrt{m_{p} m_{e}} \cdot c^{2}}$

Now it can be seen that,

$-\frac{e^{2}}{4 \pi \varepsilon_{0}\left(a_{0}\right)_{0}} \cong-\left(\frac{F_{I}\left(R_{y}\right)_{0}}{m_{e} c^{2}}\right) \cdot \frac{\sqrt{m_{p} m_{e}} \cdot c^{2}}{2}$

Thus in hydrogen atom, discrete potential energy of electron can be expressed as

$\left(E_{p o t}\right)_{0} \cong-\left(\left(\frac{F_{I}}{n^{2}}\right) \frac{\left(R_{y}\right)_{0}}{m_{e} c^{2}}\right) \cdot \frac{\sqrt{m_{p} m_{e}} \cdot c^{2}}{2}$

The major advantage of this relation is that, it constitutes the proton mass and the characteristic nuclear size. If one is willing to accept this relation as a fundamental relation in atomic physics, from relation (37)- in terms of $h_{0}$, electron's potential energy can be expressed as

$\left(E_{p o t}\right)_{0} \cong-\left(\frac{\hbar_{0} c}{G_{A} m_{e}^{2}}\right)^{2} \cdot \frac{\sqrt{m_{p} m_{e}} \cdot c^{2}}{2 n^{2}}$

Now with reference to Bohr's first postulate,

$m_{e} v r \cong n . \hbar$

At present, with in the Bohr radius

$m_{e}\left(v_{0}\right)_{0}\left(a_{0}\right)_{0} \cong \hbar_{0}$

Thus present speed of electron in the Bohr radius can be expressed as 
$\left(v_{0}\right)_{0} \cong \frac{\hbar_{0}}{m_{e}\left(a_{0}\right)_{0}} \cong \frac{1}{2} \sqrt{\frac{4 \pi \varepsilon_{0}\left(R_{y}\right)_{0} m_{p} c^{2}}{e^{2}}} \sqrt{\frac{4 \pi \varepsilon_{0}\left(R_{y}\right)_{0}^{2} F_{I}}{e^{2}}} \cdot c$

If total energy is half of the potential energy, at present, in hydrogen atom, electron's characteristic discrete total energy can be expressed as

$\left(E_{\text {total }}\right)_{0} \cong-\left(\left(\frac{F_{I}}{n^{2}}\right) \frac{\left(R_{y}\right)_{0}}{m_{e} c^{2}}\right) \cdot \frac{\sqrt{m_{p} m_{e}} \cdot c^{2}}{4}$

where $\mathrm{n}=1,2,3, \ldots$ In terms of $\mathrm{h}_{0}$

$\left(E_{\text {total }}\right)_{0} \cong-\left(\frac{\mathrm{h}_{0} c}{G_{A} m_{e}^{2}}\right)^{2} \cdot \frac{\sqrt{m_{p} m_{e}} \cdot c^{2}}{4 n^{2}}$

Please note that, from Bohr's theory of hydrogen atom, emitted photon energy is inversely proportional to $\left(\hbar_{0}^{2}\right)$ From quantum theory of light, photon energy is directly proportional to $\left(\hbar_{0}\right)$. This is a very sensitive point to be discussed in depth. At any given cosmic time,

$\left(E_{\text {total }}\right)_{t} \cong-\left(\left(\frac{F_{I}}{n^{2}}\right) \frac{\left(R_{y}\right)_{t}}{m_{e} c^{2}}\right) \cdot \frac{\sqrt{m_{p} m_{e}} \cdot c^{2}}{4}$
$\left(E_{\text {total }}\right)_{t} \cong-\left(\frac{\hbar_{t} c}{G_{A} m_{e}^{2}}\right)^{2} \cdot \frac{\sqrt{m_{p} m_{e}} \cdot c^{2}}{4 n^{2}}$

Thus it can be suggested that, $E_{\text {total }} \propto\left(R_{y}\right)_{t} \propto\left(\frac{c}{H_{t}}\right)$. Now with reference to Bohr's second postulate, at present

$\left(E_{P h o}\right)_{0} \cong\left(\frac{\hbar_{0} c}{G_{A} m_{e}^{2}}\right)^{2} \frac{\sqrt{m_{p} m_{e}} \cdot c^{2}}{4}\left(\frac{1}{n_{1}^{2}}-\frac{1}{n_{2}^{2}}\right) \cong \frac{2 \pi \hbar_{0} c}{\lambda_{0}}$.

In the past, at any galaxy, emitted photon energy can be expressed as

$\left(E_{\text {Pho }}\right)_{t} \cong\left(\frac{\hbar_{t} c}{G_{A} m_{e}^{2}}\right)^{2} \cdot \frac{\sqrt{m_{p} m_{e}} \cdot c^{2}}{4}\left(\frac{1}{n_{1}^{2}}-\frac{1}{n_{2}^{2}}\right) \cong \frac{2 \pi \hbar_{t} c}{\lambda_{G}}$

where $n_{2}>n_{1}$. Now for any quantum jump, in the past it can be shown that,

$\hbar_{t} \cong\left(\frac{1}{n_{1}^{2}}-\frac{1}{n_{2}^{2}}\right)^{-1}\left(\frac{G_{A}^{2} m_{e}^{4}}{c^{3} \sqrt{m_{p} m_{e}}}\right)\left(\frac{8 \pi}{\lambda_{G}}\right)$

Corresponding to this obtained $\hbar_{t}$, from the relation $H_{0} \hbar_{0}^{2} \cong H_{t} \hbar_{t}^{2}$ its corresponding $H_{t}$ can be estimated. From $H_{t}$ and from relation (23) corresponding CMBR energy density can be estimated. Thus for any galaxy, where $\hbar_{t}$ was playing a key role, corresponding present cosmic red shift can be expressed as

$z_{0} \cong \frac{\left(E_{P h o}\right)_{0}-\left(E_{P h o}\right)_{G}}{\left(E_{P h o}\right)_{0}} \cong 1-\left(\frac{\hbar_{t}}{\hbar_{0}}\right)^{2} \cong 1-\left(\frac{\lambda_{0}}{\lambda_{G}}\right)^{2}$

Now, approximately from relation (16), time taken by light to travel from observed galaxy to our galaxy or the age difference of our galaxy and the observed galaxy can be expressed as

$$
\Delta t \cong \frac{z_{0}}{H_{0}} \cong\left[1-\left(\frac{\hbar_{t}}{\hbar_{0}}\right)^{2}\right] \frac{1}{H_{0}} \cong\left[1-\left(\frac{\lambda_{0}}{\lambda_{G}}\right)^{2}\right] \frac{1}{H_{0}}
$$

Obtained $\Delta t$ has to be verified with other developed absolute methods of galaxy age estimation. 


\section{Light speed rotating Black Holes: The special holes}

Origin of 'rotating black hole' formation can be understood with the classical power limit $\left(c^{5} / G\right)$ and $\left(\mathrm{Mc}^{2}\right)$ within 3 steps. For any rotating celestial body assume that,

torque, $\tau \leq M c^{2}$

power, $P=\tau \omega \leq \frac{c^{5}}{G}$

thus, $\omega \leq \frac{\mathrm{c}^{3}}{\mathrm{GM}}$ and $\omega_{\max }=\frac{\mathrm{c}^{3}}{\mathrm{GM}}$

If the celestial body rotates at light speed, to have maximum angular velocity, radius should be minimum as,

$R_{\min }=\frac{c}{\omega_{\max }}=\frac{G M}{c^{2}}$

This expression is similar to the 'Schwarzschild radius' of a black hole. The only change is that coefficient 2 is missing. This is really a very interesting case. This obtained expression indicates that, to get 'light speed rotation', celestial body should have a 'minimum radius' of $G M / c^{2}$.

Clearly speaking this proposal is very simple and is entirely different from the existing concepts of General theory of relativity. It is not speaking about the gravitational collapse of stars or space-time curvature or singularity. Now this is the time to re-examine the foundations of modern black hole physics. If the concept of 'Schwarzschild radius' is believed to be true, for any rotating celestial body or black hole of rest mass (M) the critical conditions can be stated as follows.

\section{Case-1: Two statements}

1) Magnitude of 'torque' never crosses 'rest energy' .

2) Magnitude of mechanical power never crosses $\frac{1}{2}\left(c^{5} / G\right)$.

Now above relations (55) to (58) can be re-expressed as follows.

torque, $\tau \leq M c^{2}$

power, $P=\tau \omega \leq \frac{c^{5}}{2 G}$

thus, $\omega \leq \frac{\mathrm{c}^{3}}{2 \mathrm{GM}}$ and $\omega_{\max }=\frac{\mathrm{c}^{3}}{2 \mathrm{GM}}$

When the celestial body rotates at light speed, to have maximum angular velocity, radius should be minimum as,

$R_{\text {min }}=\frac{c}{\omega_{\max }}=\frac{2 G M}{c^{2}}$

Case-2: Four statements

1) Magnitude of 'kinetic energy' never crosses 'rest energy'.

2) Magnitude of 'torque' never crosses 'potential energy' and

3) Magnitude of mechanical power never crosses $\left(c^{5} / G\right)$.

4) Note that, based on the Virial theorem, potential energy is twice of kinetic energy and thus, $\tau \leq 2 M c^{2}$.

In this way in a simplified way, existence of rotating black hole concepts can be understood. Now above relations (55) to (58) can be re-expressed as follows.

torque, $\tau \leq 2 M c^{2}$

power, $P=\tau \omega \leq \frac{c^{5}}{G}$ 
thus, $\omega \leq \frac{\mathrm{c}^{3}}{2 \mathrm{GM}}$ and $\omega_{\max }=\frac{\mathrm{c}^{3}}{2 \mathrm{GM}}$

When the celestial body rotates at light speed, to have maximum angular velocity, radius should be minimum as,

$R_{\min }=\frac{c}{\omega_{\max }}=\frac{2 G M}{c^{2}}$

This expression is similar to the 'Schwarzschild radius' of a black hole and represents light speed rotation for any celestial sphere.

\section{Discussion and conclusions}

\subsection{About the cosmic geometry}

If universe constitutes so many galaxies and if each galaxy constitutes a growing and fast spinning central black hole, then considering the whole universe as a growing and light speed rotating primordial black hole may not be far from reality. Astrophysicists believe that recent observations of Super-Eddington accreting massive black holes at high redshift may provide some insight [10] on the cosmic rate of expansion. This idea directly and indirectly indicates the role of galactic central black holes in understanding the cosmic evolution. Similar to the chicken-egg problem, now the fundamental question to be answered is : which is a primordial black hole: either the universe or galaxy centre?

\subsection{About the cosmic redshift}

Whatever may the expression, the definitions of cosmic redshift seem to be ad-hoc and not absolute. With redshift concepts one may not be able to find the actual rate of cosmic expansion. From cosmology point of view, the real picture of cosmic rate of expansion can be understood from the 'rate of decreasing' cosmic thermal energy density.

\subsection{About the proposed new concepts}

Parallel to the existing modern mathematical concepts like 'higher dimensions' and 'dark energy' if one is willing to consider the proposed new concepts, definitely a unified model of cosmology and quantum physics can be developed. But it may take some time to understand and follow them. From atomic physics point of view, the real picture of cosmic rate of expansion can be understood only from the increasing Planck's constant or the increasing 'inverse' fine structure ratio or the increasing characteristic nuclear unit radius.

\section{Acknowledgements}

The first author is indebted to professor K. V. Krishna Murthy, Chairman, Institute of Scientific Research on Vedas (ISERVE), Hyderabad, India and Shri K. V. R. S. Murthy, former scientist IICT (CSIR) Govt. of India, Director, Research and Development, I-SERVE, for their valuable guidance and great support in developing this subject.

\section{References}

[1] Hawking S.W. A brief history of time. Bantam Dell publishing group.1998

[2] J.K. Webb et al. Indications of a spatial variation of the fine structure constant. Physical Review letters, 107 (19) 2011

[3] Hubble E. P, A relation between distance and radial velocity among extra-galactic nebulae, PNAS, 1929, vol. 15, 1929, pp.168-173.

[4] Hubble, E.P, The 200-inch telescope and some problems it may solve. PASP, 59, pp153-167, 1947.

[5] David Gross, Einstein and the search for Unification. Current science, Vol. 89, No. 12, 25 Dec 2005.

[6] Narlikar J.V. Direct Particle Formulation of Mach's Principle. Einstein Studies, vol. 6: Mach's Principle: From Newton's Bucket to Quantum Gravity, pp. 250-261 ® 1995 Birkhauser Boston, Inc. Printed in the United States

[7] U. V. S. Seshavatharam, S. Lakshminarayana, B.V.S.T. Sai. Is red shift - an index of galactic 'atomic light emission' mechanism? International Journal of Physics, 2013, Vol. 1, No. 3, 49-64

[8] Joshua Frieman, Michael Turner and Dragan Huterer, Dark Energy and the Accelerating Universe. Ann. Rev. Astron. Astrophys. 46: 85-432, 2008.

[9] Geiger H and Marsden E. On a diffuse reaction of the particles. Proc. Roy. Soc., Ser. A 82: 495-500, 1909.

[10] Jian-Min Wang, Pu Du, David Valls-Gabaud, Chen Hu, and Hagai Netzer. Super-Eddington Accreting Massive Black Holes as Long-Lived Cosmological Standards, Physical Review Letters, Vol 110 Issue 8, 2013. DOI:10.1103/PhysRevLett.110.081301 